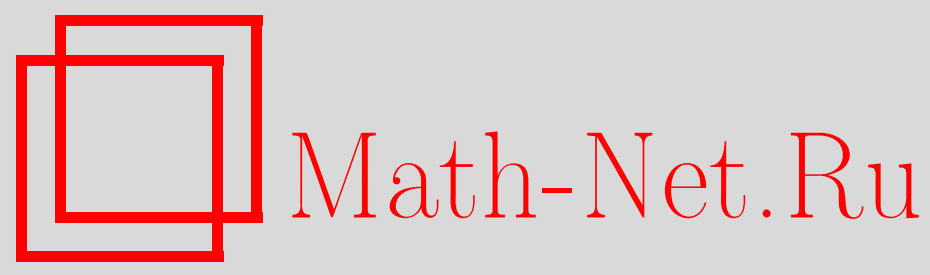

В. П. Маслов, Об одном новом способе вторичного квантования фермионов, Матем. заметки, 1997, том 61, выпуск 4, 627-629

DOI: https://doi.org/10.4213/mzm1541

Использование Общероссийского математического портала Math-Net.Ru подразумевает, что вы прочитали и согласны с пользовательским соглашением http://www . mathnet.ru/rus/agreement

Параметры загрузки:

IP: 35.174 .16 .151

26 апреля 2023 г., 13:42:44

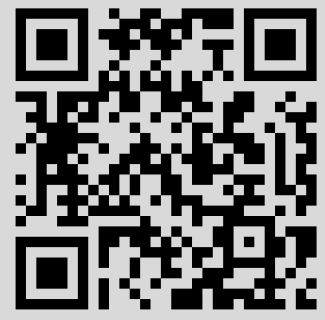




\section{ОБ ОДНОМ НОВОМ СПОСОБЕ \\ ВТОРИЧНОГО КВАНТОВАНИЯ ФЕРМИОНОВ}

\section{В.П. Маслов}

Рассматривается задача об $N$ фермионных частицах, описьваемая уравнением Шрёдингера

$$
\begin{aligned}
E \psi\left(x_{1}, \ldots, x_{N}\right)= & \sum_{l=1}^{N}\left(-\frac{1}{2} \Delta_{x_{l}}+U\left(x_{l}\right)\right) \psi\left(x_{1}, \ldots, x_{N}\right) \\
& +\frac{\varepsilon}{2} \sum_{1 \leqslant l \neq m \leqslant N} V\left(x_{l}, x_{m}\right) \psi\left(x_{1}, \ldots, x_{N}\right),
\end{aligned}
$$

где $x_{i} \in \mathbb{R}^{d}, \varepsilon \in \mathbb{R}, \psi\left(x_{1}, \ldots, x_{N}\right)$ - антисимметричная функция. Пусть $k$ - целое число такое, что $0 \leqslant k \leqslant N$ и $N-k=2 M, M$ - целое. Любой антисимметричной функции $\psi(x)$ соответствует функция

$$
\rho\left(x_{1}, i_{1} ; \ldots ; x_{k}, i_{k} ; z_{1}, w_{1} ; \ldots ; z_{M}, w_{M}\right)=\psi\left(x_{1}, \ldots, x_{k}, z_{1}, w_{1}, \ldots, z_{M}, w_{M}\right) \cdot \epsilon_{i_{1}, \ldots, i_{k}},
$$

где $\forall l=\overline{1, k}, i_{l}=\overline{1, k}$, а $\epsilon_{i_{1}, \ldots, i_{k}}$ - абсолютньй антисимметричньй тензор ранга $k$, симметричная относительно своих аргументов $x_{l}, i_{l}$ и $x_{m}, i_{m}$, а также $z_{p}, w_{p}$ и $z_{q}, w_{q}$, удовлетворяющая уравнению

$$
\begin{aligned}
(E & \left.-\sum_{j=1}^{k} \lambda_{j}\right) \rho \\
= & \sum_{l=1}^{k}\left(-\frac{1}{2} \Delta_{x_{l}}+U\left(x_{l}\right)-\lambda_{i_{l}}\right) \rho+\sum_{p=1}^{M}\left(-\frac{1}{2} \Delta_{z_{p}}+U\left(z_{p}\right)-\frac{1}{2} \Delta_{w_{p}}+U\left(w_{p}\right)\right) \rho \\
& +\left\{\frac{\varepsilon}{2} \sum_{1 \leqslant l \neq l^{\prime} \leqslant k} V\left(x_{l}, x_{l^{\prime}}\right)+\frac{\varepsilon}{2} \sum_{1 \leqslant p \neq p^{\prime} \leqslant M}\left(V\left(z_{p}, z_{p^{\prime}}\right)+V\left(w_{p}, w_{p^{\prime}}\right)\right)\right. \\
& \left.+\varepsilon \sum_{p, p^{\prime}=1}^{M} V\left(z_{p}, w_{p^{\prime}}\right)+\sum_{l=1}^{k} \sum_{p=1}^{M}\left(V\left(x_{l}, z_{p}\right)+V\left(x_{l}, w_{p}\right)\right)\right\} \cdot \underset{\substack{x_{1}, \ldots, x_{k}, z_{1}, \ldots, w_{M} \\
\operatorname{Assymm}}}{\operatorname{Asymm} \rho, i_{k},}
\end{aligned}
$$

где $\lambda_{j}, j=\overline{1, k},-$ некоторые действительные числа, $\rho=\rho\left(x_{1}, i_{1} ; \ldots ; x_{k}, i_{k} ; z_{1}, w_{1} ; \ldots\right.$; $\left.z_{M}, w_{M}\right)$

$$
\begin{aligned}
& \underset{\operatorname{ssymm}_{k}, z_{1}, \ldots, w_{M}}{ } \psi\left(x_{1}, \ldots, x_{k}, z_{1}, w_{1}, \ldots, z_{M}, w_{M}\right) \\
& =\frac{1}{(k+2 M) !} \sum_{\sigma}(-1)^{\operatorname{sign} \sigma} P_{\sigma} \psi\left(x_{1}, \ldots, w_{M}\right),
\end{aligned}
$$

где $P_{\sigma}-$ перестановка аргументов функции $\psi$, и

$$
\underset{i_{1}, \ldots, i_{k}}{\operatorname{Asymm}} f\left(i_{1}, \ldots, i_{k}\right)=\frac{1}{k !} \sum_{\widetilde{\sigma}}(-1)^{\operatorname{sign} \widetilde{\sigma}} U_{\widetilde{\sigma}} f\left(i_{1}, \ldots, i_{k}\right),
$$


$U_{\widetilde{\sigma}}$ - перестановка аргументов $f$. Назовем $x_{1}, i_{1}, \ldots, x_{k}, i_{k}$ простыл типом переменных а а $z_{1}, w_{1}, \ldots, z_{M}, w_{M}-$ парным мином. Функции $\rho$ ставится в соответствие вектор пространства Фока [1] вида

$$
\begin{aligned}
\widetilde{\rho}=\sum_{i_{1}, \ldots, i_{k}=1}^{k} \int & \cdots \int d x_{1} \cdots d x_{k} d z_{1} d w_{1} \cdots d z_{M} d w_{M} \rho\left(x_{1}, i_{1}, \ldots ; z_{1}, w_{1}, \ldots\right) \\
& \times \widehat{b}^{+}\left(x_{1}, i_{1}\right) \cdots \widehat{b}^{+}\left(x_{k}, i_{k}\right) \widehat{A}^{+}\left(z_{1}, w_{1}\right) \cdots \widehat{A}^{+}\left(z_{M}, w_{M}\right) \Psi_{0}
\end{aligned}
$$

где $\widehat{b}^{ \pm}(x, i), \widehat{A}^{ \pm}(z, w)$ - операторы, удовлетворяющие коммутационным соотношениям

$$
\begin{aligned}
&\left.\widehat{b}^{ \pm}(x, i), \widehat{b}^{ \pm}(y, j)\right]=\left[\widehat{A}^{ \pm}(z, w), \widehat{A}^{ \pm}\left(z^{\prime}, w^{\prime}\right)\right]=\left[\widehat{b}^{ \pm}(x, i), \widehat{A}^{ \pm}(z, w)\right] \\
&=\left[\widehat{b}^{ \pm}(x, i), \widehat{A}^{\mp}(z, w)\right]=0, \\
& {\left[\widehat{b}^{-}(x, i), \widehat{b}^{+}(y, j)\right]=\delta_{i j} \delta(x-y), \quad\left[\widehat{A}^{-}(z, w), \widehat{A}^{+}\left(z^{\prime}, w^{\prime}\right)\right]=\delta\left(z-z^{\prime}\right) \cdot \delta\left(w-w^{\prime}\right), }
\end{aligned}
$$

а вектор $\Psi_{0}$ - вакуумный вектор пространства Фока. Вектор $\widetilde{\rho}$ удовлетворяет уравнению

$$
\widetilde{E} \widetilde{\rho}=\widehat{\mathscr{H}} \widetilde{\rho}, \quad \text { где } \quad \widetilde{E}=E-\sum_{j=1}^{k} \lambda_{j},
$$

оператор $\widehat{\mathscr{H}}$ имеет следующий вид:

$$
\begin{aligned}
\widehat{\mathscr{H}}= & \sum_{i=1}^{k} \int d x \widehat{b}^{+}(x, i)\left(-\frac{1}{2} \Delta_{x}+U(x)-\lambda_{i}\right) \widehat{b}^{-}(x, i) \\
& +\iint d z d w \widehat{A}^{+}(z, w)\left(-\frac{1}{2} \Delta_{z}+U(z)-\frac{1}{2} \Delta_{w}+U(w)\right) \widehat{A}^{-}(z, w) \\
& + \\
& \left\{\frac{\varepsilon}{2} \sum_{i, j=1}^{k} \iint d x d y V(x, y) \widehat{b}^{+}(x, i) \widehat{b}^{+}(y, j) \widehat{b}^{-}(y, j) \widehat{b}^{-}(x, i)\right. \\
+ & \frac{\varepsilon}{2} \iiint \int d z d w d z^{\prime} d w^{\prime}\left(V\left(z, z^{\prime}\right)+V\left(w, w^{\prime}\right)\right) \\
& \times \widehat{A}^{+}(z, w) \widehat{A}^{+}\left(z^{\prime}, w^{\prime}\right) \widehat{A}^{-}\left(z^{\prime}, w^{\prime}\right) \widehat{A}^{-}(z, w) \\
+ & \varepsilon \iint d z d w V(z, w) \widehat{A}^{+}(z, w) \widehat{A}^{-}(z, w) \\
+ & \varepsilon \iiint \int d z d w d z^{\prime} d w^{\prime} V\left(z, w^{\prime}\right) \widehat{A}^{+}(z, w) \widehat{A}^{+}\left(z^{\prime}, w^{\prime}\right) \widehat{A}^{-}\left(z^{\prime}, w^{\prime}\right) \widehat{A}^{-}(z, w) \\
+ & \varepsilon \sum_{i=1}^{k} \iiint d x d z d w(V(x, z)+V(x, w)) \\
& \left.\times \widehat{b}^{+}(x, i) \widehat{b}^{-}(x, i) \widehat{A}^{+}(z, w) \widehat{A}^{-}(z, w)\right\} \sum_{m=0}^{\infty} \widehat{\sigma}_{m} \widehat{P}_{m},
\end{aligned}
$$


где операторы $\widehat{\sigma}_{m}$ и $\widehat{P}_{m}$ имеют следующий вид:

$$
\begin{aligned}
& \widehat{P}_{m}=\frac{1}{m ! k !}\left(\sum_{i=1}^{k} \int d x \widehat{b}^{+}(x, i) \widehat{1}^{-}(x, i)\right)^{k} \\
& \times \exp \left(-\sum_{j=1}^{k} \int d y \widehat{b}^{+}(y, j) \widehat{b}^{-}(y, j)\right)\left(\iint d z d w \widehat{A}^{+}(z, w) \widehat{A}^{-}(z, w)\right)^{m} \\
& \times \exp \left(-\iint d z^{\prime} d w^{\prime} \widehat{A}^{+}\left(z^{\prime}, w^{\prime}\right) \widehat{A}^{-}\left(z^{\prime}, w^{\prime}\right)\right), \\
& \widehat{\sigma}_{m}=\frac{1}{k ! m !} \sum_{i_{1}, \ldots, i_{k}=1}^{k} \int \cdots \int d x_{1} \cdots d x_{k} d z_{1} d w_{1} \cdots d z_{m} d w_{m} \\
& \times \widehat{b}^{+}\left(x_{1}, i_{1}\right) \cdots \widehat{b}^{+}\left(x_{k}, i_{k}\right) \widehat{A}^{+}\left(z_{1}, w_{1}\right) \cdots \widehat{A}^{+}\left(z_{m}, w_{m}\right) \\
& \times \underset{x_{1}, \ldots, x_{k}, z_{1}, w_{1}, \ldots, z_{m}, w_{m}}{\operatorname{Asymm}} \underset{i_{1}, \ldots, i_{k}}{\operatorname{Asymm}} \widehat{A}^{-}\left(z_{m}, w_{m}\right) \cdots \widehat{A}^{-}\left(z_{1}, w_{1}\right) \widehat{b}^{-}\left(x_{k}, i_{k}\right) \cdots \widehat{b}^{-}\left(x_{1}, i_{1}\right) .
\end{aligned}
$$

K оператору (3) для нахождения его собственных значений и собственных функций $(2)$ применима теория комплексного ростка [2]-[4].

Аналогично можно провести рассмотрение квантовой электродинамики. В этой теории состояние описьвается вектором $\psi_{N_{+}, N_{-}}\left(\vec{p}_{1} i_{1}, \ldots, \vec{p}_{N_{+}} i_{N_{+}} ; \vec{k}_{1} j_{1}, \ldots, \vec{k}_{N_{-}} j_{N_{-}}\right)$, где $N_{+}$- число позитронов, $N_{-}$- число электронов, $\vec{p}_{l} i_{l}$ и $\vec{k}_{m} j_{m}$ - импульсная и спиновая переменные соответственно позитронов и электронов. Функция $\psi_{N_{+}, N_{-}}(\vec{p} i, \vec{k} j)$ антисимметрична относительно перестановок $\vec{p}_{m} i_{m}$ и $\vec{p}_{l} i_{l}$, а также $\vec{k}_{m} j_{m}$ и $\vec{k}_{l} j_{l}$. Гамильтониан теории таков, что сохраняется полньй заряд $Z=N_{+}-N_{-}$. Поэтому задача "симметризуется" аналогично (1) относительно двух типов переменных: простого по электронньм переменным от $\vec{p}_{1} i_{1}$ до $\vec{p}_{|Z|} i_{|Z|}$ (если $Z \leqslant 0$; если $Z>0$, то аналогично, но по позитронньм переменным) и парного по парам электрон-позитрон от $\vec{p}_{|Z|+1} i_{|Z|+1}, \vec{k}_{1} j_{1}$ до $\vec{p}_{N_{-}} i_{N_{-}}, \vec{k}_{N_{+}} j_{N_{+}}$.

Московский государственный университет

Поступило

им. М.В. Ломоносова

05.03.97

\section{СПИСОК ЦИТИРОВАННОЙ ЛИТЕРАТУРЫ}

1. Березин Ф. А. Метод вторичного квантования. М.: Наука, 1986. 2. Маслов В. П. Комплексный метод ВКБ в нелинейных уравнениях. М.: Наука, 1977. 3. Маслов В.П., Шведов О. Ю. // ТМф. 1994. Т. 98. № 2. С. 266-288. 4. Maslov V. P. // Russian J. Math. Phys. 1995. V. 3. № 3. P. 401-406. 